\title{
IMPLEMENTATION OF NEW NOVEL DESIGN REAL TIME ABNORMAL MOTION DETECTION SCHEME FOR VIDEO SURVEILLANCE
}

\author{
Mr. S. P. SANTHOSHKUMAR, \\ Assistant Professor, Department of CSE, \\ Rathinam Technical Campus, Coimbatore, Tamil Nadu, India \\ Dr. H. Lilly Beaulah \\ Professor, Head of the Department, Department of CSE, \\ Mahendra College of Engineering, Salem, Tamil Nadu, India
}

\begin{abstract}
In this paper is clarified about Video reconnaissance frameworks create tremendous measures of information for capacity and show. Long haul human checking of the obtained video is unrealistic and incapable. Programmed unusual movement recognition framework which can adequately draw in administrator consideration and trigger account is in this way the way to effective video reconnaissance in unique scenes, for example, airplane terminal terminals. This paper exhibits a novel answer for continuous unusual movement identification. The proposed strategy is appropriate for current video-reconnaissance designs, where restricted figuring power is accessible close to the camera for pressure and correspondence. In this venture, we propose a novel and precise way to deal with movement discovery for the programmed video reconnaissance framework. Our technique accomplishes finish identification of moving items by including three noteworthy proposed modules: a foundation displaying (BM) module, a caution trigger (AT) module, and a protest extraction (OE) module. For our proposed BM module, a special two-stage foundation coordinating technique is performed utilizing quick coordinating taken after by precise coordinating keeping in mind the end goal to deliver ideal foundation pixels for the foundation show. Next, our proposed AT module wipes out the superfluous examination of the whole foundation district, permitting the resulting $\mathrm{OE}$ module to just process squares containing moving articles. At long last, the $\mathrm{OE}$ module shapes the twofold protest discovery cover with a specific end goal to accomplish exceptionally entire identification of moving items. The location comes about created by our proposed (PRO) strategy were both subjectively and quantitatively broke down through visual assessment and for precision, alongside correlations with the outcomes delivered by other best in class strategies. The calculation utilizes the full scale square movement vectors that are created regardless as a major aspect of the video pressure process. Movement highlights are gotten from the movement vectors. The factual circulation of these highlights amid typical action
\end{abstract}

is evaluated via preparing. At the operational stage, impossible movement include values demonstrate unusual movement.

Keywords: video surveillance system, Background Modeling (BM) module, An Alarm Trigger (AT) Module and an Object Extraction (OE) module. Macro-Block Motion Vectors

\section{INTRODUCTION}

The most well known approach to execute movement location calculation is by actualizing the picture division and picture subtraction strategies of PC vision. The greater part of the calculation first fragments the frontal area moving items from the foundation picture. To do this, they would need to take an arrangement of pictures with no movement as a matter of course to introduce the foundation picture. This foundation picture would be refreshed thusly to give a continuous situation where changes to the foundation are taken into contemplations. For instance, when a moving item halted, it would then be a piece of the foundation picture.

Obviously, the closer view articles can be procured by utilizing basic number juggling utilizing picture subtraction. The aftereffect of the subtraction procedures where pixels having a place with the present picture are subtracted by the comparing pixels out of sight picture or the other way around would acquire the closer view moving items. Subsequent to acquiring the forefront protests, the concentration for locale of intrigue is set to these frontal area questions rather than the entire picture. In this manner additionally picture handling is performed just on these districts of intrigue. One of the vital strides here is to remove imperative highlights of the moving items to perceive the protest. Be that as it may, perceiving 
the question is past this examination subject as this module of the undertaking is to give the articles and the contributions to the protest arrangement motor.

An exceptionally well known approach for identifying faces is the Viola Jones strategy which makes utilization of a supported course of straightforward highlights and a basic picture portrayal to take into account extremely fast face discovery. While it is conceivable to track a face by identifying it in each new casing this prompts a ton of jitter in the yield and is likewise extremely wasteful. All things considered this strategy for following isn't regularly utilized , anyway the Viola Jones finder is frequently utilized by other following strategies to at first find the face.

There are different ways to deal with recognition; for instance utilizes a mix of skin shading investigation and movement location taken after by a help vector machine to identify faces in a video succession. A few different strategies are specified in anyway the Viola-Jones approach is by all accounts supported over these today in light of its speed.

\section{MOVING EDGE DETECTION}

Presenting edge identifiers helps in acquiring a reasonable photo of the moving items. Ramesh, Rangachar and Brian and Milan, Vaclav, Roger both expressed in their content that utilizing edge indicators consolidating with the diverse pictures will give a superior result for movement recognition by coming more than a few downsides. There are many edge locators calculations being presented in the picture preparing field. Generally all content on picture handling would cover the themes anxious identification.

There are additionally many web references that portrays convolution strategies that might be connected to edge discovery calculations too.

Ramesh, Rangachar and Brian recognized 3 stages in edge discovery calculations which are:

$>$ Filtering

$>$ Enhancement

$>$ Detection

"Many edge finders have been produced over the most recent two decades." cited from some first subsidiaries administrator veils utilized for edge identifiers are Roberts administrator, Sobel administrator, Prewitt administrator. Others utilize second subsidiaries administrator veils, for example, Laplacian administrator. A few uses Gaussian edge location techniques, a well known strategy which utilizes the vigilant edge indicator.

Foundation does not generally remain the same. In this manner to enhance the most fundamental calculation examined in the past subject, we influence B into a 1D to space. For instance, let $t 2$ signifies the time that a foundation is refreshed. A refreshing capacity signified by $\mathrm{Fn}[\mathrm{B}(\mathrm{t} 2)]$ alludes to the capacity to refresh the foundation picture $\mathrm{B}$ at time $\mathrm{t} 2$.

Accordingly, in the wake of playing out the underlying count, the closer view is presently extricated from this new foundation meant by $\mathrm{B}(\mathrm{t} 2)$. Hence changing the condition (1) into;

$\mathrm{P}[\mathrm{F}(\mathrm{t})]=\mathrm{P}[\mathrm{I}(\mathrm{t})]-\mathrm{P}[\mathrm{B}(\mathrm{t} 2)](2)$

Where $\mathrm{B}(\mathrm{t} 2)=\mathrm{Fn}[\mathrm{B}]$ meaning $\mathrm{B}(\mathrm{t} 2)$ is figured by a refreshing capacity that is performed on the past foundation. One path is by utilizing information gathered from the casings and plays out some figuring on the foundation picture.

One case is to bring or record down every one of the pixels of the considerable number of edges before time $t 2$. From that point forward, essentially totals them up and ascertain the normal esteem and refresh the foundation B to get another foundation $\mathrm{B}(\mathrm{t} 2)$ with this normal esteem. $\mathrm{Be}$ that as it may, there have been numerous approaches to refresh the foundation.

The upside of their technique is that there capacity, for example, Fn[B] talked about above should be possible in each edge as they utilize an aggregate capacity to decide the consequence of the subtraction. Milan, Vaclav, Roger likewise introduced a comparable gathering diverse picture strategy signified as "combined distinctive picture" in their content.

Ming and Tim utilized a Gaussian blend display in light of the RGB shading space for keeping up a foundation for movement discovery. Their work is successful when connected to open air movement location frameworks where it is quick because of enlightenment changes for instance brightening changes emerging from moving mists.

Moore likewise actualized a Gaussian model for keeping up a foundation for movement recognition. As indicated by his compositions, the Gaussian models refresh can be founded on either the K-Means or Expectation Maximization (EM) calculations. In Ming and Tim paper, they executed the EM calculation.

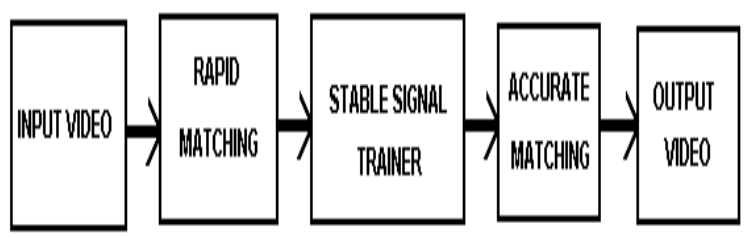

Fig. 1. Back Ground Subtraction Accomplish

The presently executed technique for foundation subtraction achieves its target by subtracting every pixel of the approaching video outline from the foundation display, 
hence producing a flat out distinction. It at that point applies an edge to get the double protests discovery veil. Limit choice is a basic task and can be led by an assortment of already inquired about strategies. In spite of the fact that the at present actualized foundation subtraction technique is helpful for usage, the clamor resistance in the video outline depends on the decided edge.

Functionalities, for example, question order, following, conduct, and recognizable proof are then performed on the areas where moving items have been identified. The computational expenses of customary forefront examination strategies are normally generally costly for the video reconnaissance frameworks in view of the conventional optical stream usage.

For more exact movement location configuration, frontal area investigation is constantly required for the most well known foundation subtraction technique keeping in mind the end goal to accomplish the examination of the movement data. Concerning foundation support, the pixellevel procedures and area level procedures ought to be unmistakably composed away from plain sight subtraction approach.

This is on the grounds that pixel-level procedures can deal with the adjustment to changing foundation at every pixel autonomously without pixels aggregate perception, and locale level process can refine the crude order of the pixel-level concerning interpixel connections

In this paper is indicates BM module, a one of a kind two-stage foundation coordinating methodology is performed utilizing quick coordinating taken after by precise coordinating keeping in mind the end goal to create ideal foundation pixels for the foundation demonstrate.

In this area, we show a novel movement location approach for static-camera observation situations. Our approach accomplishes finish identification of moving items and includes three proposed modules: a foundation demonstrating (BM) module, an AT module, and a protest extraction $(\mathrm{OE})$ module. At first, the proposed BM module outlines a one of a kind two-stage foundation coordinating system utilizing fast coordinating taken after by precise coordinating keeping in mind the end goal to deliver ideal foundation pixels for the foundation display.

So as to definitely diminish the computational multifaceted nature of the movement discovery process, we propose utilizing an AT module. This module comprises of a novel square based entropy assessment strategy created for the work of square hopefuls, after which the doubtlessly moving items inside the movement squares are resolved in view of square based morphological disintegration and widening tasks.

The AT module wipes out the superfluous examination of the whole foundation locale, enabling the OE module to just process squares containing moving articles. As the last advance of the procedure, the proposed OE module looks at each square which may potentially contain moving articles keeping in mind the end goal to produce the twofold question recognition cover. This is refined by using the reasonable limit esteem, which is then connected naturally through our proposed powerful edge choice calculation.

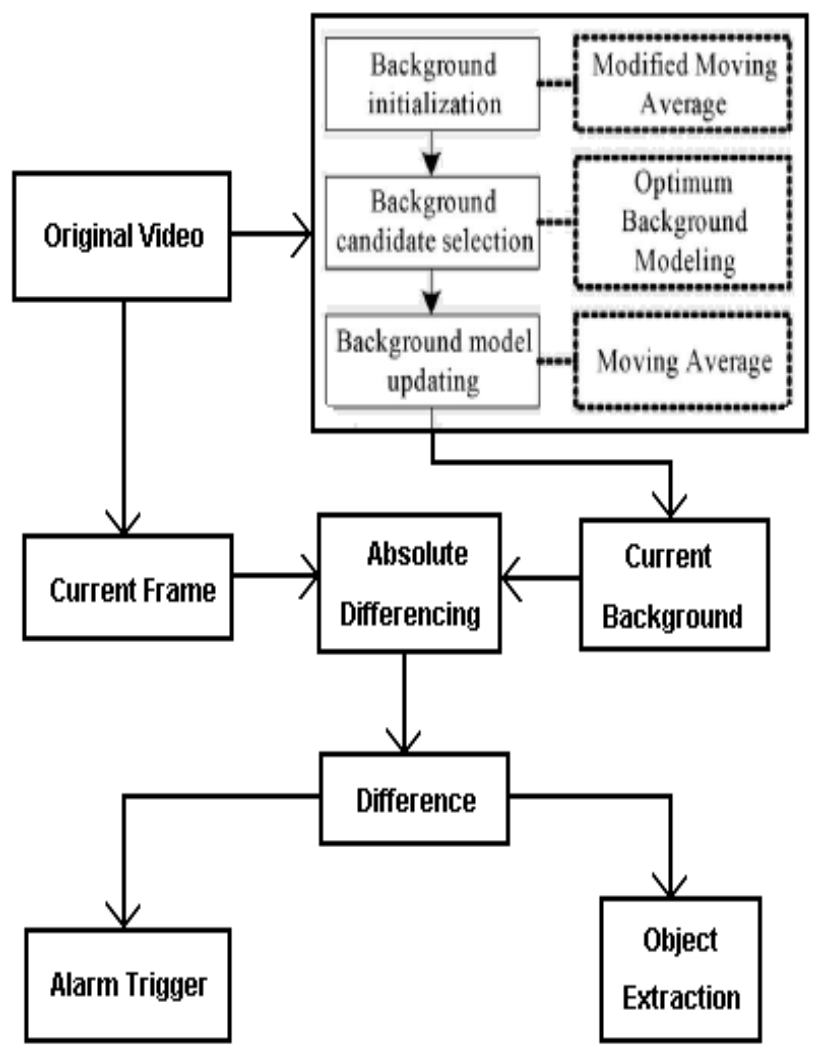

Fig 2. Modified Accomplish Model

AT module dispenses with the pointless examination of the whole foundation area, permitting the resulting OE module to just process squares containing moving articles. At long last, the OE module shapes the twofold question recognition cover to accomplish exceedingly entire discovery of moving items.

\section{A. Novel Background Subtraction Method}

This task displays a novel foundation subtraction technique which creates a foundation demonstrate utilizing the chose appropriate foundation hopefuls. At that point, using a caution trigger (AT) module, it distinguishes the pixels of moving articles inside the locales resolved to altogether highlight objects.

The association of the proposed (PRO) strategy is as per the following. 
1) A two-stage foundation coordinating strategy is utilized to choose reasonable foundation contender for age of a refreshed foundation demonstrate.

2) A square based entropy assessment with morphological tasks is directed through an activated square based caution module.

3) Production of movement discovery is finished through the programmed edge choice calculation.

\section{BACKGROUND MODELING}

\section{A. Initial Background Model:}

The altered moving normal (MMA) is utilized to figure the normal of edges 1 through $\mathrm{K}$ for the underlying foundation show generation. For every pixel $(x, y)$, the comparing estimation of the present foundation demonstrate $\mathrm{Bt}(\mathrm{x}, \mathrm{y})$ is ascertained.

B. Optimum Background Model:

For the ideal foundation pixel estimation in the video arrangement, the fundamental target of OBM is to remove the steady flag of the approaching casing in the video succession. As can be seen, the flag removed by OBM is more steady than those of the un-separated flag in alternate edges.

The structure of OBM comprises of the accompanying advances:

1) Immediate assurance of foundation hopefuls through the fast coordinating technique.

2) Use of the steady flag mentor so as to give a measure of transient action of the pixels inside the arrangement of foundation competitors;

3) Determination of the ideal foundation pixels by means of the exact coordinating strategy.

\section{Rapid matching}

This system is utilized to rapidly locate an extraordinary amount of foundation hopefuls by deciding if their particular pixel esteems for the approaching video outline $\operatorname{It}(\mathrm{x}, \mathrm{y})$ are equivalent to the relating pixel estimations of the past video outline It $-1(\mathrm{x}, \mathrm{y})$. In the event that the qualities relate, it demonstrates great competitor determination for the accompanying stable flag mentor.

\section{Stable signal trainer}

All pixels from the arrangement of foundation applicants chose by means of the quick coordinating technique are prepared through the steady flag trainer.The stable flag coach is communicated as takes after:

$$
M_{t}(x, y)=\left\{\begin{array}{lc}
M_{t}(x, y)+p, & \text { if } I_{t}(x, y)>M_{t-1}(x, y) \\
M_{t}(x, y)-p, & \text { if } I_{t}(x, y)<M_{t-1}(x, y)
\end{array}\right.
$$

\begin{tabular}{|c|c|c|c|c|c|c|c|c|c|c|c|}
\hline 12 & 0.55 & 0.23 & 0 & 1.32 & 0.53 & 0.23 & 0.89 & 0.79 & 0.32 & 0 & 1.44 \\
\hline 1.23 & 0.12 & 0 & 4.60 & a81 & 0 & 0 & 0 & 0.20 & 0.70 & 0.58 & 0.83 \\
\hline 23 & 0 & 0.96 & 4.47 & $0 . \pi$ & 1.60 & 0 & 0.93 & 0 & 0 & 0.77 & 0 \\
\hline 32 & 0 & 5.16 & 4.85 & 4.67 & $\rho$ & 0 & 1.13 & 0 & 0.12 & 0 & 0 \\
\hline 0 & 0.58 & 4.11 & 390 & 406 & 0.59 & 0 & 0 & 0.32 & 0.12 & 0.77 & 0.27 \\
\hline 0.43 & 0.78 & 4.59 & 399 & 390 & 1.53 & 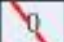 & 0 & 0.34 & 0.40 & 1.80 & 0 \\
\hline 1.00 & 0.92 & 5.06 & 495 & 4.97 & 1.45 & 0. & & & & & 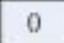 \\
\hline 0 & 0.32 & 5.02 & 5.41 & 5.55 & 0.66 & & & & & & 1.19 \\
\hline 0.23 & 0 & 2.67 & 5.25 & 3.09 & 0 & & & & & & 0 \\
\hline 0 & 0 & 2.69 & 5.46 & 2.79 & 0 & & & & & & 0 \\
\hline 0 & 0 & 2.36 & 5.19 & 2.64 & 0 & & & & & & 0 \\
\hline 0 & 0 & 3.20 & 5.46 & 3.10 & 0 & 0 & 0 & 0 & 0 & 0 & 0 \\
\hline 0 & 0 & 2.36 & 4.22 & 436 & 3.58 & 3.22 & 3.10 & 2.34 & 1.19 & 0.12 & 0 \\
\hline 0 & 0 & 0 & 0.40 & 0 & 0 & 0 & 0 & 0.20 & 0.73 & 0.76 & 0.20 \\
\hline
\end{tabular}

Fig 3 Block-Based Entropy Value in a Video Frame

Where $\operatorname{Mt}(\mathrm{x}, \mathrm{y})$ is the relating pixel inside the latest arrangement of foundation hopefuls, $\mathrm{Mt}-1(\mathrm{x}, \mathrm{y})$ is the comparing pixel inside the past arrangement of foundation competitors, and $\mathrm{p}$ speaks to the genuine esteem.

\section{E. Accurate matching}

Each light-dim pixel of the foundation hopeful is prepared to the dull dark pixel by utilizing the steady flag mentor. The ideal of these are then decided from the dull dark pixels when the pixels of $\operatorname{Mt}(\mathrm{x}, \mathrm{y})$ are equivalent to $\mathrm{It}(\mathrm{x}, \mathrm{y})$. They are spoken to here in dark.

\section{F. Alarms Trigger Module}

After the foundation demonstrate is created by means of the BM method at each edge, the supreme distinction $t(x$, y) is produced by without a doubt the differential estimation between the refreshed foundation display $\mathrm{Bt}(\mathrm{x}$, $\mathrm{y})$ and current approaching video outline $\operatorname{It}(\mathrm{x}, \mathrm{y})$.

With a specific end goal to essentially quicken the accompanying OE module, we suggest that the AT module be included a stepwise system including novel square based entropy assessment taken after by square based morphological tasks.

\section{BLOCK - BASED MORPHOLIGAL EROSION}

Recognition of every conceivable movement square competitor is proficient by the proposed square based entropy assessment. Disposal of a portion of the identified foundation squares and finished movement squares is then performed by means of the square based morphological disintegration and expansion activities.

In this way, the yield flag of the foundation display must be more steady keeping in mind the end goal to 
separate between the trembling signs of moving items and those delivered by the foundation. At the point when contrasted with other best in class strategies, it ends up evident that the yield foundation flag of our proposed foundation show displays less difference.

Notwithstanding foundation display assessment, the subjective movement identification comes about acquired through the proposed (PRO) strategy were likewise contrasted and those gotten through other best in class techniques. To get estimations of quantitative exactness, distinctive measurements were performed on a few video groupings; these included Recall, Precision, and Similarity.

\section{VIDEO SURVILEILIANCE}

On the off chance that we consider video in the least complex of terms, video reconnaissance started with basic shut circuit TV checking (CCTV). As ahead of schedule as 1965 , there were squeeze reports in different nations over the world proposing police utilization of observation cameras out in the open spots. At the point when videocassette recorders hit the market, video observation turned out to be extremely mainstream. Simple innovation utilizing taped video-tape accounts implied observation could be saved on tape as proof. A total simple videoreconnaissance framework comprised of a camera, screen, and VCR.

The old tube camera was just helpful in sunshine, and the VCR could just store eight long stretches of film, best case scenario. The disadvantage was that sooner or later, proprietors and representatives of such a framework would end up self-satisfied and not change the tapes every day or the tapes would destroy following quite a while of being re-utilized. There was likewise the issue of account around evening time or in low light. While the idea was great, the innovation hadn't yet topped. The subsequent stage was the Charged Coupled Device camera (CCD), which utilized microchip PC innovation. In the 1990's video reconnaissance made awesome walks in common sense by the presentation of advanced multiplexing. At the point when computerized multiplexer units ended up reasonable, it reformed the reconnaissance business by empowering recording on a few cameras without a moment's delay.

Three key components expedited the famous utilization of the advanced video recorder, They are

$>$ The headway in pressure capacity, enabling more data to be put away on a hard drive. (Round-theclock reconnaissance creates a great deal of data.)

$>$ The cost of a hard drive, which has dropped significantly as of late.

$>$ The stockpiling limit of a hard drive, which has expanded significantly as of late.
Computerized video observation seemed well and good as the cost of advanced account dropped with the PC transformation. Instead of changing tapes day by day, the client could dependably record multi month of reconnaissance on hard drive.

The pictures recorded carefully were such a great amount of clearer than the regularly grainy pictures recorded with simple that acknowledgment was instantly enhanced for recognizable proof purposes. Carefully put away pictures can likewise be upgraded in different ways (include light, change hues, turn around highly contrasting) to make vital conclusions. With tape, what you see is the thing that you get.

\section{A. Requirement of Video Surveillance}

While it is imperative to comprehend the different spots video reconnaissance can be utilized it is likewise critical to asses the dangers associated with the insurance of a specific thing. In the ongoing years, as an ever increasing number of things, for example, workmanship are picking up significance, the costs of such things are additionally experiencing the rooftop. In this way, innovation has come in the bleeding edge for assurance and observation of such products and things. While evaluating hazard one of your sources of info ought to be burglary measurements. The accompanying are the measurements of burglaries in spots, for example, shops, living arrangements and in broad daylight puts in our nation, India in a specific year. Of the 50 revealed robberies in a single year, the breakage of burglaries can be appeared as the accompanying.

$>$ Display Cases 19

$>$ Open shows 10

$>$ Pictures 04

$>$ Other shows 02

$>$ At night 06

$>$ From stores 02

$>$ Long timescale 04

$>$ Other 03

This implies despite the fact that innovation has enhanced drastically in the previous couple of decades, it has still far to go. It is plainly observed from the insights that despite the fact that the focal point of security reconnaissance is on picking up prove against wrongdoings and robberies, the manner of thinking should 


\section{International Journal of Engineering Applied Sciences and Technology, 2019 \\ Vol. 3, Issue 12, ISSN No. 2455-2143, Pages 92-98 \\ Published Online April 2019 in IJEAST (http://www.ijeast.com)}

change to halting burglaries and violations while they are in advance.

\section{B. Motion Detection in Live Video Stream}

At the point when all is said and done, observation frameworks ought to be an impression of this present reality we live in. As individuals turn out to be increasingly security canny, they will request genuine insurance for their property. The new computerized video frameworks should raise that security to another level.

They should influence the clients to rest easy. Drive away a couple of troublemakers. Furthermore, the individuals who do attempt to beat the framework should confront a far more serious danger of getting captured. Subsequently, the new computerized video observation frameworks ought to have the capacity to give a high conviction that all is good. The significant serenity must be accomplished when the individual is guaranteed that he will be educated of any robberies of his property while they are in advance.

He would likewise feel more secure in the event that he can be ensured that the observation framework that he uses won't just give him prove against the culprits yet in addition endeavor to prevent the robberies from occurring in any case. Along these lines, to accomplish such sort of security Motion Detection in the live video stream is actualized. The movement location frameworks won't just screen the regions of intrigue yet will likewise keep a functioning post for any movement being delivered.

\section{ACQUISITION SETUP}

The Matlab programming dialect is utilized to store information as grids. Accordingly Matlab can furnish speedy interface with information frameworks. The product accommodates outline obtaining from equipment gadgets, for example, web cams or computerized cameras as long as the gadgets are accurately instated by the software engineer. Along these lines, so as to permit brisk setup with the picture procurement gadgets, Matlab Function index gives a large group of predefined works by which the client can ask about the different diverse gadgets right now associated and afterward setup the required gadget with Matlab so it can get and store information at run time.

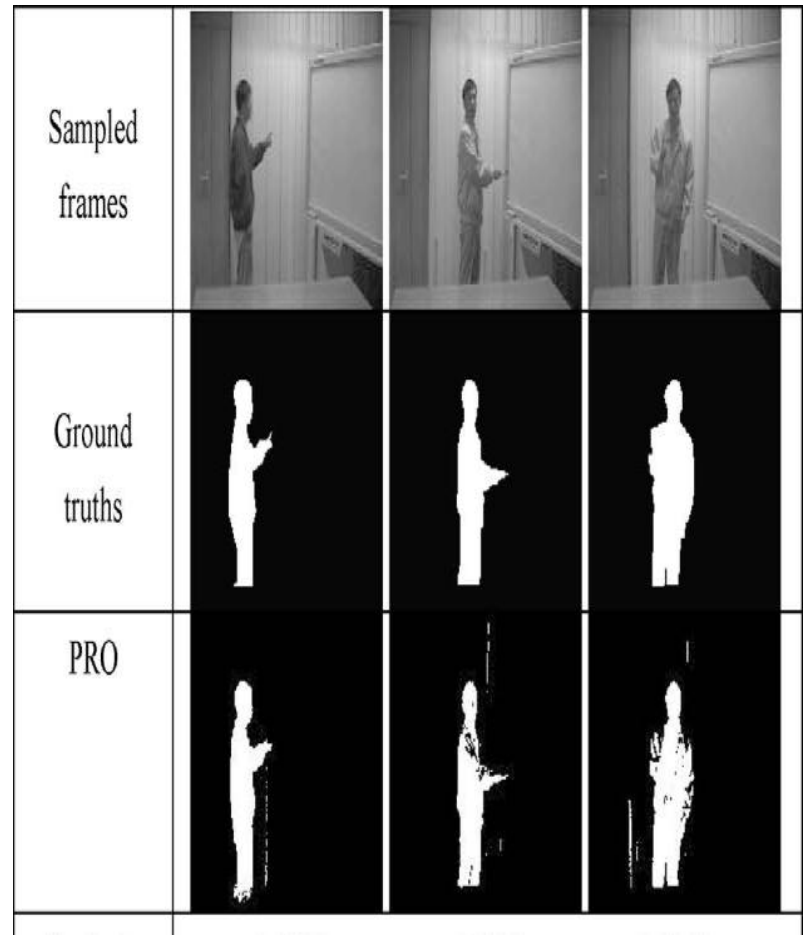

Fig 4 Tracking Image 1

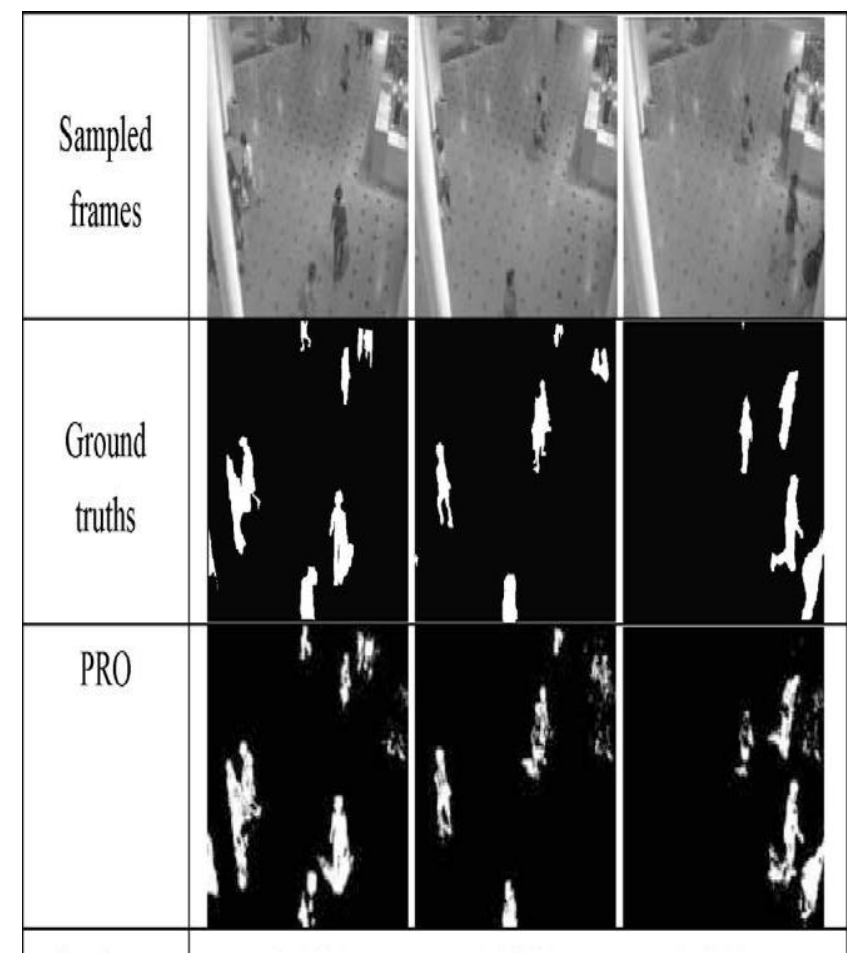

Fig 5 Tracking Image 2 


\section{A. Motion Detection Algorithm}

The Matlab interface enables the client to characterize the charges to be performed at the run time. Once the client setup of the video source is finished the calculation becomes possibly the most important factor. The calculation is worked to exploit the quality of Matlab i.e. to store information as a type of frameworks.

The edges procured are put away in the Matlab catalog as grid in which every component of the framework contains data about the pixel estimation of the picture at a specific area. In this manner, the pixel esteems are put away in the workspace as a lattice where each component of the network relates to an individual pixel esteem.

Since Matlab considers every framework as one vast gathering of qualities rather than a cluster of individual qualities it is fundamentally speedier in breaking down and handling the picture information. The calculation subsequently checks each casing being gained by the gadget with the beforehand procured casing and checks for the contrast between the aggregate estimations of each casing. A limit level is set by the client with which the distinction of qualities is thought about. In the event that the distinction surpasses the edge esteem the movement is said to be recognized in the video stream.

\section{CONCLUSION}

The strategy can distinguish moving individual track them and give one of a kind tag to precisely following people in indoor reconnaissance video stream acquired from a static camera with troublesome scene properties including brightening changes and tackle real impediment issue with bring down storage room prerequisites. A versatile following calculation with imperatives of frontal area locales is planned keeping in mind the end goal to enhance the productivity and heartiness of movement following. The proposed question extraction module distinguished the pixels of moving items inside the activated caution area to shape the moving articles veil. After a brilliant foundation show was created, the AT module wiped out the pointless examination of the whole foundation locale and lessened the computational unpredictability for the consequent movement identification stage. In this manner, the proposed AT module can viably encourage the execution of the whole movement location process, while requiring minimal computational cost by the essential tasks in the proposed AT chain.

\section{REFERENCES:}

1. Nehme, M.A.; Khoury, W.; Yameen, B.; Al-Alaoui, M.A., "Real time color based motion detection and tracking", Proc. ISSPIT 2003, 3rd IEEE International Symposium on Signal Processing and Information Technology, 2003, 14-17 Dec. 2003 , pp. 696 - 700, 1417 Dec. 2003.

2. Josué A. Hernández-García, Héctor Pérez-Meana and Mariko Nakano- Miyatake, "Video Motion Detection Using the Algorithm of Discrimination and the Hamming Distance", Lecture Notes in Computer Science, SpringerVerlag, Germany.

3. H.A.M. El_Salamony, H.F. Ali, and A.A. Darweesh, "3D Human Body Motion Detection and Tracking in Video", Proc. Acta Press.

4. M.Kavitha, V.Sivachidambaranathan, "Comparison of Different Control Techniques for Interleaved DC-DC Converter," International Journal of Power Electronics and Drive System, Vol.9(2), pp.641-647, June 2018

5. Jayaprakash, S. and Murugan, A. Solar Based DC-DC Converters for Shunt Motor Applications. Research Journal of Pharmaceutical Biological and Chemical Sciences 7 (4) (2016) 930-933.

6. Song, Y.,"A perceptual approach to human motion detection and labeling", $\mathrm{PhD}$ thesis, California Institute of Technology, 2003.

7. Yilmaz, A., M. Shah, "Contour Based Object Tracking with Occlusion Handling in Video Acquired Using Mobile Cameras", Proc. IEEE Transactions on Pattern Analysis and Machine Intelligence, 2005.

8. M.Kavitha, V.Sivachidambaranathan, "Transformer less Inverter using Unipolar Sinusoidal Pulse Width Modulation Technique for Grid Connected Photovoltaic Power System", International Journal of Applied Engineering Research, Vol.10, no.2,pp. 3089$3100,2015$.

9. Thien Huynh, et al, "NIC: A Robust Background Extraction Algorithm for Foreground Detection in Dynamic Scenes" IEEE Transactions on Circuits and Systems for Video Technology volume 27 issue 7, 2017 Pp 1478 - 1490

10.A.Murugan, V.Ramakrishnan, "Modeling and control of GSO method based on HPFC using an interconnected hybrid power generation systems" Journal of Advanced Research in Dynamical and Control Systems Volume 15 Special Issue 2017 pp: 780-786.

11. Samuele Salti, et al., "Synergistic Change Detection and Tracking" IEEE Transactions on Circuits and Systems for Video Technology volume 25 issue 4, 2015 PP- 609622

12. Chun-Yu Chen; Yu Shao, "Crowd Escape Behavior Detection and Localization Based on Divergent Centers" IEEE Sensors Journalvolume 15 Issue 42015 pp: 2431 2439

13.Jae Hyuk Shin; Boreom Lee; Kwang Suk Park, "Detection of Abnormal Living Patterns for Elderly Living Alone Using Support Vector Data Description" EEE Transactions on Information Technology in Biomedicine volume 15 Issue 32011 pp- 438-448 